\title{
INDOOR AIR QUALITY MANAGEMENT USING RASPBERRY PI AND GRAPHLAB
}

\author{
Somdeep Acharyya ${ }^{1}$, Sayan Hazra ${ }^{2}$, Atanu Pal ${ }^{3}$, Nabarun Sengupta ${ }^{4}$ \\ ${ }^{1}$ Student, Department of Computer Science and Engineering, University Institute of Technology, \\ Burdwan University, West Bengal, India \\ ${ }^{2}$ Student, Department of Electrical Engineering, University Institute of Technology, \\ Burdwan University, West Bengal, India \\ ${ }^{3}$ Student, Department of Applied Electronics and Instrumentation Engineering, University Institute of Technology, \\ Burdwan University, West Bengal, India \\ ${ }^{4}$ Nabarun Sengupta, Department of Electronics and Communication Engineering, University Institute of Technology, \\ Burdwan University, West Bengal, India
}

\begin{abstract}
Indoor air pollution is a matter of serious concern at present times. Indoor air pollution or indoor air quality degradation is becoming an issue of discussion among scientists and environmentalists in today's days. As human beings generally spend most of their time indoors, having good indoor air quality and its real time information is critical for maintaining human health and productivity. According to the EPA, indoor air even in centrally air conditioned buildings is more polluted than outdoor air, due to improperly maintained ventilation systems. Hence it's an utmost necessity for human beings. Many reports have come out showing how much harmful is Indoor air quality pollution and the bad effects and diseases caused by such deterioration in the indoor air quality. Many solutions have been devised to tackle this air abuse and reduce its effects, also several methods of analyzing the quality of air and further monitoring through specialized sensors, the quality of air inside our household rooms like bedrooms or drawing rooms, even in classrooms and halls. But each of the other works have their own limitations and restraints, some are gathering data only from the classrooms or hall rooms. Nowhere there was any implementation of such measuring system in a bedroom, drawing room. In this research work, we have proposed a quick and easy solution of monitoring the air quality using dedicated sensors by connecting them to a microcontroller. The entire setup is desired to be placed inside classrooms full of students as well as in household rooms having people in them, at various times of the day with different temperature and humidity conditions. The data collected from the classrooms and drawing rooms or bedrooms under several environmental instances are to be uploaded to a server, from where it would be retrieved into our machines. After this collection of data, this data to be analyzed using some machine learning tool to invigilate the attributes of the air present in the rooms, namely the concentration of $\mathrm{CO}_{2}, \mathrm{CO}$, the quantity of dust particles and their relative variations with changes in temperature or humidity or the number of persons present inside the room. Thus we have developed (1) a prototype to measure the indoor air pollutant concentration (2) a wireless sensor network architecture with a microcontroller (3) a data analyzing algorithm using a sophisticated but popular machine learning tool. Advantages of this prototype is that it was of very low cost and measurement was effective, also the presentation of the data was not at all robust but easy to view and monitor.
\end{abstract}

Keywords: Raspberry pi, $\mathrm{CO}_{2}$, CO, dust, Sensors, temperature and humidity, IAQM, Zigbee, Python, Graphlab

\section{INTRODUCTION}

Air quality is one of important measures to be closely monitored in real time for today's urban ecosystems, because air quality has a major influence on the health, safety, productivity and comfort of people. With growing transportation and population density, increasing global warming and sudden climate change, the air quality has frequently below the environmental quality standards. Many countries have developed their own infrastructure to monitor and provide alerts to their citizens. However it is presumed nowadays that besides the atmospheric air quality, indoor air quality is deteriorating day by day with enormous use of Air conditioners and other gadgets, also due to the excessive use of various devices of particular usage. It is reported that this degradation of air quality is extremely harmful for human lives as several diseases are introduced to the body due to this indoor air pollution. Even it is predicted that air borne diseases are occurring in much due to atmospheric air pollution, but because of the bad quality of air inside the so called safe places such as rooms like classrooms, bedrooms, drawing rooms and more. According to a report in the National Air quality Standards, the major important factors which deform the air quality are as such: $\mathrm{CO}_{2}, \mathrm{CO}$, Dust Particles, $\mathrm{SO}_{2}$, Ozone and Radon. The $\mathrm{CO}_{2}, \mathrm{CO}$ are present in minute quantity in the atmosphere, Dust particles are also present in the air, more due to the human activities, So2 and some other oxides of Nitrogen and Sulphur also are associated with the air pollution. Ozone plays a major role in affecting the air quality even inside the room. A very rare element Radon is also a part of this game, which is released in the air from the damped walls of old buildings and from 
cemented floors even. Though in minute quantity, still the presence of Radon in the air present inside the rooms is harmful for the persons living inside them. Dust particles play a major role in the pollution and are extremely harmful. Many studies have shown that exposure to atmospheric particulate matter (PM) is associated with adverse health impacts such as heart and respiratory diseases. In this research, we have developed an air quality sensor system using $\mathrm{CO}_{2}, \mathrm{CO}$, Dust sensors. We have chosen only these gases as these are the major pollutants that affect the indoor air quality. Along with these, we have used a Temperature and Humidity sensor to view the changes that take place in the concentration of $\mathrm{CO}_{2}, \mathrm{CO}$, dust in the air with the changes in temperature, humidity or the number of people present inside the rooms. Our challenges are to:

Getting involved in participatory air quality monitoring to collect useful data to interpret the degree of pollution inside the rooms. These involve providing with:

1>Low-cost and low-power measurement hardware suitable for measurements of indoor air quality.

2>Unobtrusive and user-friendly data acquisition and processing and observation tool, a machine learning tool.

$3>$ Support in gathering high quality data.

To measure the urban air pollution we needed to setup the system in the streets or in railway stations or in busy public pacifies. However this will have a big disadvantage. If gas sensors are placed on vehicles running on the streets, the gas sensors can be moved or dislocated easily at random. At some frequency, every sensor runs with other sensors and they share a single location. Since several types of sensors are used in the network at the same time, the attributes or the characteristics of the measurement error are different. Some of the sensor outputs are lower than real gas concentration and the others are higher than real concentration, although they are exposed to the same gas concentration at the same place.

\section{RELATED WORKS}

This section summarizes the prior work and efforts for monitoring indoor air quality using sensors and then wirelessly monitoring accordingly. In reference[3] provides an integrated sensing node or WSN System for monitoring build upon a Raspberry Pi architecture that experimented at various places such as church, classrooms and bedrooms whereas reference[5] integrates the use of portable AQS and monitoring using smartphone on Arduino architecture which use AQS and a zone-based proximity detection method for collaborative sensing, which saves energy and enables data sharing among users but uses only $\mathrm{C} 02$ sensor similar to smartphone sensor designed in [7], whereas Air quality is defined by 7 factors which is being using by reference[1] in Arduino platform only using Wi-Fi communication and [11] uses IOT and wireless sensors.

Real time indoor sensors are being used in most of the references for indoor in some cases such as [4], [5], [8], and [12] whereas [6] designed for urban Air Quality monitoring. Most of them uses an Arduino and auto calibration sensors. Reference [3], [4] produces a feedback alert for any abrupt change of values, whereas reference [5], [6] takes real time action based on the values from the sensors. [4] Has a problem of issues such as spectrum availability detection, interference mitigation and spectrum sharing. Reference [7] to improve accuracy and energy efficiency, MAQS incorporates three novel techniques an accurate temporal ngram augmented Bayesian room localization method that requires few Wi-Fi fingerprints an air exchange rate based IA sensing method, which measures general IAQ using only $\mathrm{CO}_{2}$ sensors and a zone-based proximity detection method for collaborative sensing, which saves energy and enables data sharing among users. Others use Wi-Fi module, whereas reference [2],[9], [10] uses Zigbee devices for communication between sensors and an interface for monitoring.

\section{SETUP}

The IAQ assessment system deals in the detection and analysis of Indoor Air Quality. In fact, Indoor Air Quality is measured in the built environment, provides a continuous stream of information for monitoring the air conditions inside the room and provides a platform for informed decision making.

The prototype has two layers, a Raspberry Pi based system associated with $\mathrm{CO}$ sensor, $\mathrm{CO}_{2}$ sensor, Temperature and Humidity sensor and dust sensor and a monitoring systemGraph Lab Create with Jupyter Notebook. This study describes the IAQ system, developed by the authors, which aims to ensure autonomously and accurately the IAQ monitoring in different indoor places such as bedrooms, drawing rooms, and classrooms. The system consists of a low cost indoor air quality monitoring wireless sensor system, developed using Raspberry PI, Wi-Fi modules and micro sensors, for storage and availability of monitoring data on the server in real time and analyzes the changes in the concentration of particles and compounds with temperature and humidity.

We have placed the system in the air conditioned as well as non AC classrooms of University Institute of Technology, Burdwan University, at different times of the day, at such a places where ample amount of student activity is available and flow of air is also sufficient. We have put the setup there ensuring there is proper variation in $\mathrm{CO}_{2}$ and $\mathrm{CO}$ concentration over time. We have also placed the system in bedrooms at daytime and night for when (1) the room was empty (2) there were people working in the room (3) people were sleeping at night.

In order to achieve this objective a comprehensive review of the literature of journal articles, conference papers and edited volumes. Given that IAQ sensing is still in formative stages and not yet been realized, it was necessary to consider a wide range of sources for a comprehensive review of the topic. The IAQ is still in a conceptual state and the field is very dynamic at this point, reviewing only journal articles that make a specific contribution to the IAQ would yield only a very limited review. 


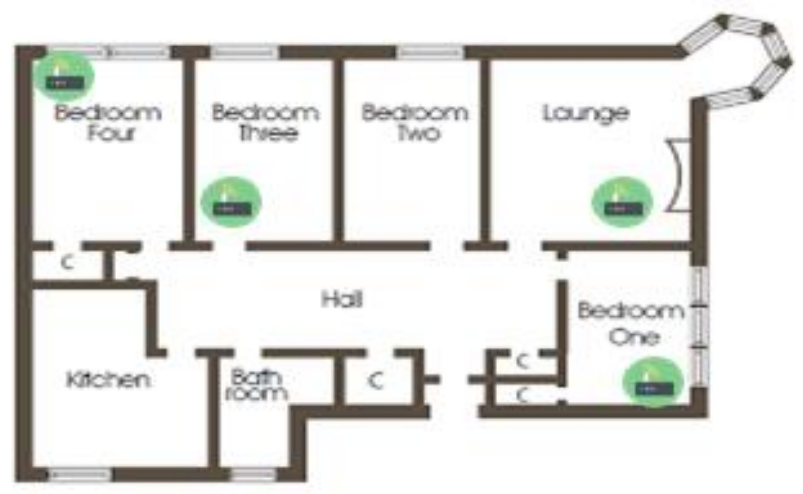

Fig-1: shows implementation of IAQM kit in household. The symbol 1 represents the kit.

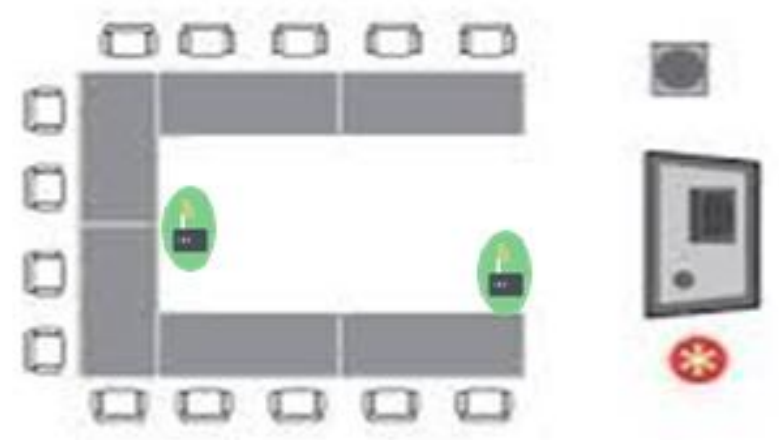

Fig-2: shows implementation of IAQM kit in classroom.

Each sensor is connected to a ZigBee radio transceiver i.e., the XBee module. There is a user application program on each sensor node, which handles sampling data from sensors. The XBee module on the base station is configured as coordinator and the XBee modules on the sensor nodes are configured as routers. To access the sensor nodes and the data from the outside world, a web application is developed on the base station. A web application is developed to provide users the data that is collected form the sensors to our local machines for the purpose of analysis using Graphlab.

\section{HARDWARE SPECIFICATIONS}

The IAQ sensors are built using the RaspberryPi a mini computer that has all the features of a computer processor. The operating system used is Raspbian and the Linux core is Debian. The firmware of the Sensor kit was implemented using the SSH Putty and the commands and codes for the sensors was given in a command terminal using basic Linux commands for Debian core.

A brief description of each sensor and components is given below:-

1>MQ7 Sensor: A highly sensitive, stable and long life Carbon Monoxide(CO) sensor with a range of $10 \mathrm{ppm}-$ $500 \mathrm{ppm}$ with an operating voltage of $5 \mathrm{~V} \pm 0.1$ and works well in temperature range of $-20^{\circ} \mathrm{C}$ to $50^{\circ} \mathrm{C}$. Humidity Range- $(55 \pm 5 \%$ RH). Requires manual calibration. It require a preheat time of 48 hours.

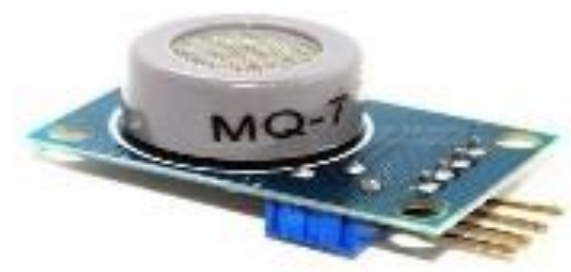

Fig-3: MQ7 sensor.

2>MQ135 Sensor: We are using this sensor for collecting the amount of $\mathrm{CO}_{2}$ in air in ppm. A high sensitivity and wide range and fast response gas sensor which can detect NH3, NOx, Benzene and Carbon Dioxide $\left(\mathrm{CO}_{2}\right)$ with a range of $10-1000 \mathrm{ppm}$. Circuit voltage $5 \mathrm{~V} \pm 0.1$ and works well at a temperature range of $-10^{\circ} \mathrm{C}$ to $45^{\circ} \mathrm{C}$. Requires manual calibration. It can collect data in $65 \% \pm 5 \%$ humidity.

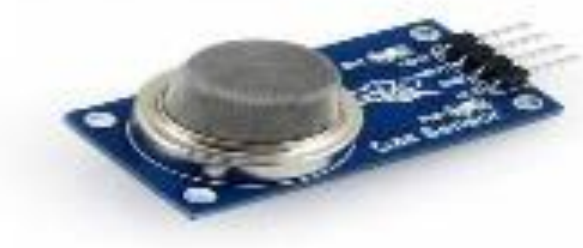

Fig-4:MQ135 sensor.

3>GP2Y1010AU0F Sensor: A dust sensor by optical sensing system. It detects the reflected light of dust in the air and is very sensitive to dust. The working temperature is $10^{\circ} \mathrm{C}$ to $+65^{\circ} \mathrm{C}$ and the working voltage is $-0.3 \mathrm{~V}$ to $7 \mathrm{~V}$. Requires manual calibration.

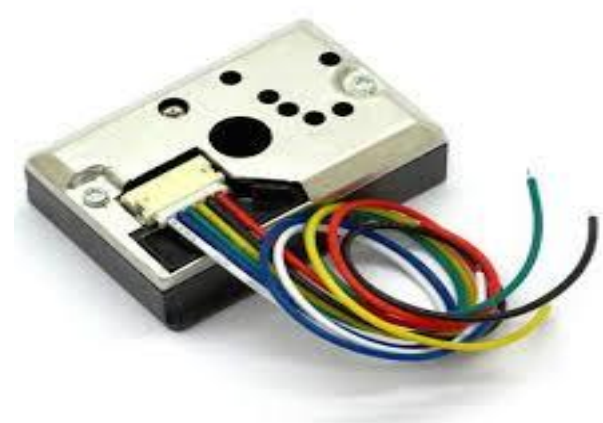

Fig-5:GP2Y1010AU0F sensor.

4>DHT11 Sensor: A digital temperature and humidity sensor, containing a calibrated digital signal output of the temperature and humidity. The sensor includes a resistive sense of wet components and an NTC temperature measurement device and connected with a high performance 8 bit microcontroller. Temperature range: $25^{\circ} \mathrm{C} \pm 2^{\circ} \mathrm{C}$.It requires a power supply of $3.5 \mathrm{~V}-5.5 \mathrm{~V} \mathrm{DC}$. 


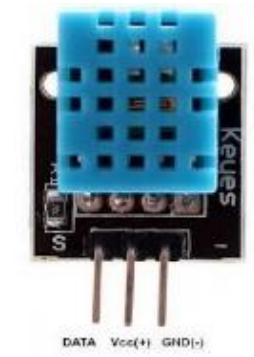

Fig-6:DHT11 sensor.

\section{SOFTWARE IMPLEMENTATION}

After the collection of data from the server to our local machine, we are converting the file into csv format. Python is used as the programming language for the entire purpose of analysis of data. We are using Jupyter Notebook for the programming, along with GraphLab Create developed by Turi Company. Jupyter Notebook App is a server-client application that allows editing and running notebook documents, i.e. ipynb files via a web browser. Jupyter notebook has a notebook kernel which is a "computational engine" that executes the code contained in a Notebook Document. An ipython kernel referenced in this module executes python code. We are also using GraphLab Create which is a graph based, high performance, distributed computation framework written in $\mathrm{C}++$. It is an open source project using an Apache license. The GraphLab framework is a parallel programming abstraction targeted for sparse iterative graph algorithms and it provides a high level programming interface, allowing a rapid deployment of distribute machine learning algorithms. For the installment of Jupyter Notebook, Anaconda is must in any machine. So we have also installed Anaconda.

We have developed an ipynb file where the python code is written, and a $\mathrm{C}$ program to initiate the ipynb file. For the browser required for the Jupyter Notebook, we have used Google Chrome. The ipynb file, the csv files which contains all the data, the $\mathrm{C}$ program all are kept in the same directory in which Anaconda is located. This is extremely important for the correct execution of the system. Another thing that was initially a limitation that the csv file did not have the column names specified (what values in what columns represented which parameter). So we separately added column names to each and every csv file containing data using python commands in Jupyter Notebook.

\section{ANALYSIS AND RESULTS}

The AQM Sensor kit collects all the transfers via the communication device to the server. Samples of data were taken from rooms containing variable number of people were taken and recorded and varied according to temperature changes.

1) The system was initially placed in the Computer Science Lab (air conditioned) in University Institute of Technology, Burdwan University during a lab session at the noon time for about 1 hour and 30 minutes. The lab was filled with pupils and there was a continuous stream of air flow as well.
2> The system was again operated at evening in another classroom in the same institution in which the room was empty for the first 20 minutes and then it was occupied with students. Data was collected from that room for about 2 hours.

3> The IAQM kit was again used in two bedrooms, one at the noon, another in the same room in evening, both instances had people activity; while the last data collection was done in another room at midnight with people sleeping in it

Thus, these were the instances under which data was collected and this data was sent to the server via the communication system associated with Raspberry pi.

Then graphical analysis is done for all the data for Carbon Monoxide (CO), Carbon Dioxide $\left(\mathrm{CO}_{2}\right)$ and dust particles with respect to time, temperature and humidity. The graphical display of the environment factors allows a greater perception of the behavior of the monitored parameter than numerical display format.

In the ipynb file that contained the python code os package and graphlab package was imported to convert the file to csv format and start graphlab analysis respectively. On executing the entire code together by running all the cells, we could start the Graphlab view in a Google chrome tab, through which we could view the data and their relative variations in Scatter plot format or in Bar graph format or in Heat maps. We can also calculate the mean, median, regression coefficients and other statistical parameters by using the Graphlab software.

The following Figs show the results that were obtained from Graphlab from the data collected from the classrooms of the institute.

A sample of the code used is shown over here:

\# coding: utf-8

\# In[49]:import graphlab\# $\operatorname{In}[50]:$ import os\# $\operatorname{In}[51]:$ file="text.doc"\# $\quad \operatorname{In}[52]: f d=$ open(file, $" r ") \#$ $\operatorname{In}[53]: \mathrm{m}=\mathrm{fd} \cdot \operatorname{read}() \#$

In[54]:data=graphlab.SFrame.read_csv(m)\#

$\operatorname{In}[55]: \mathrm{g}=$ data.show ()$\# \quad \operatorname{In}[56]:$ sqft_model = graphlab.linear_regression.create(data, target='CO2', features=['Index'],validation_set=None)\# In[57]:print sqft_model\# In[58]:print sqft_model.evaluate(data)\# In[59]:sqft_model.get('coefficients')\#_In[60]:print sqft_model.show() 


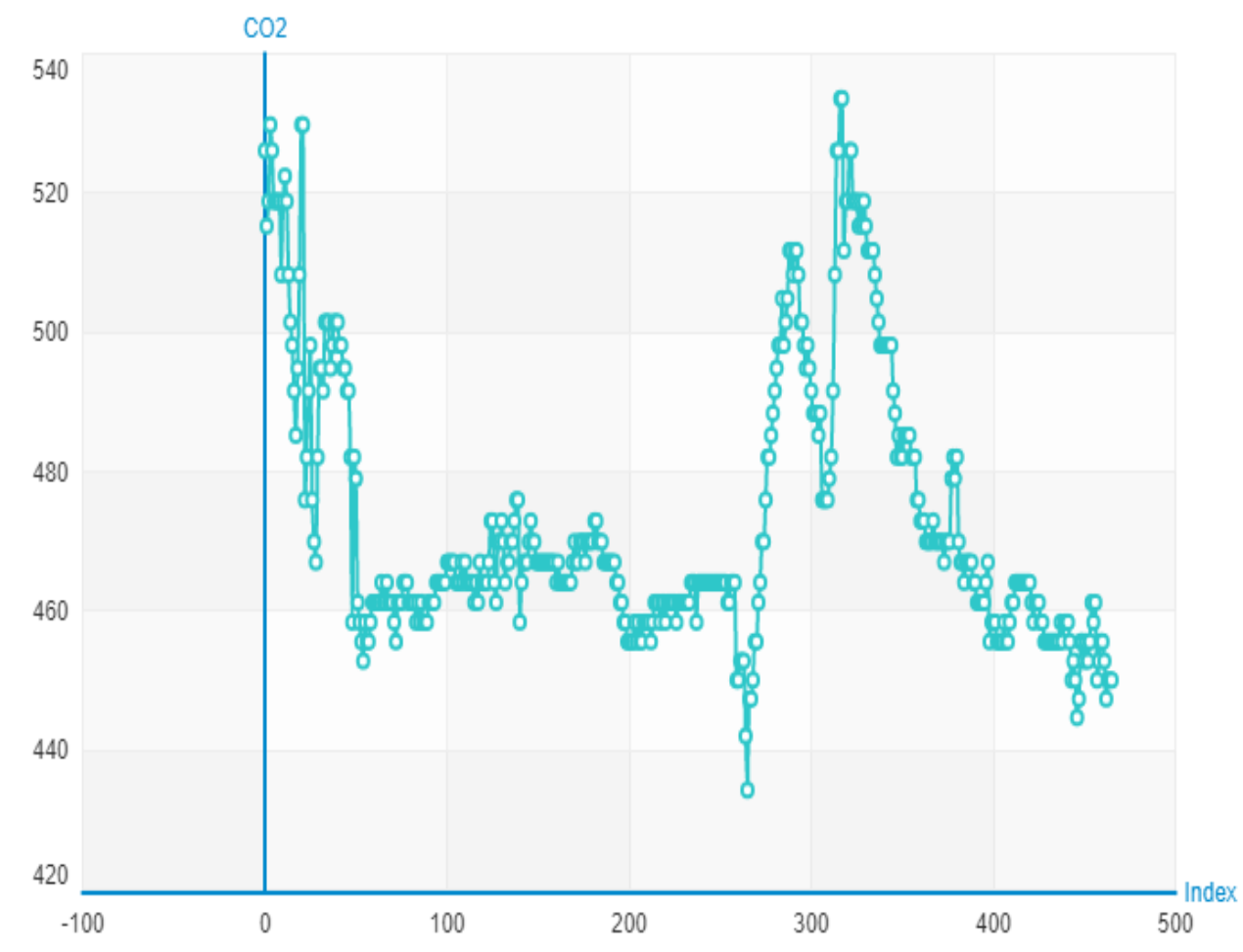

Fig-7: shows the variation of $\mathrm{CO}_{2}$ with Time (Index).

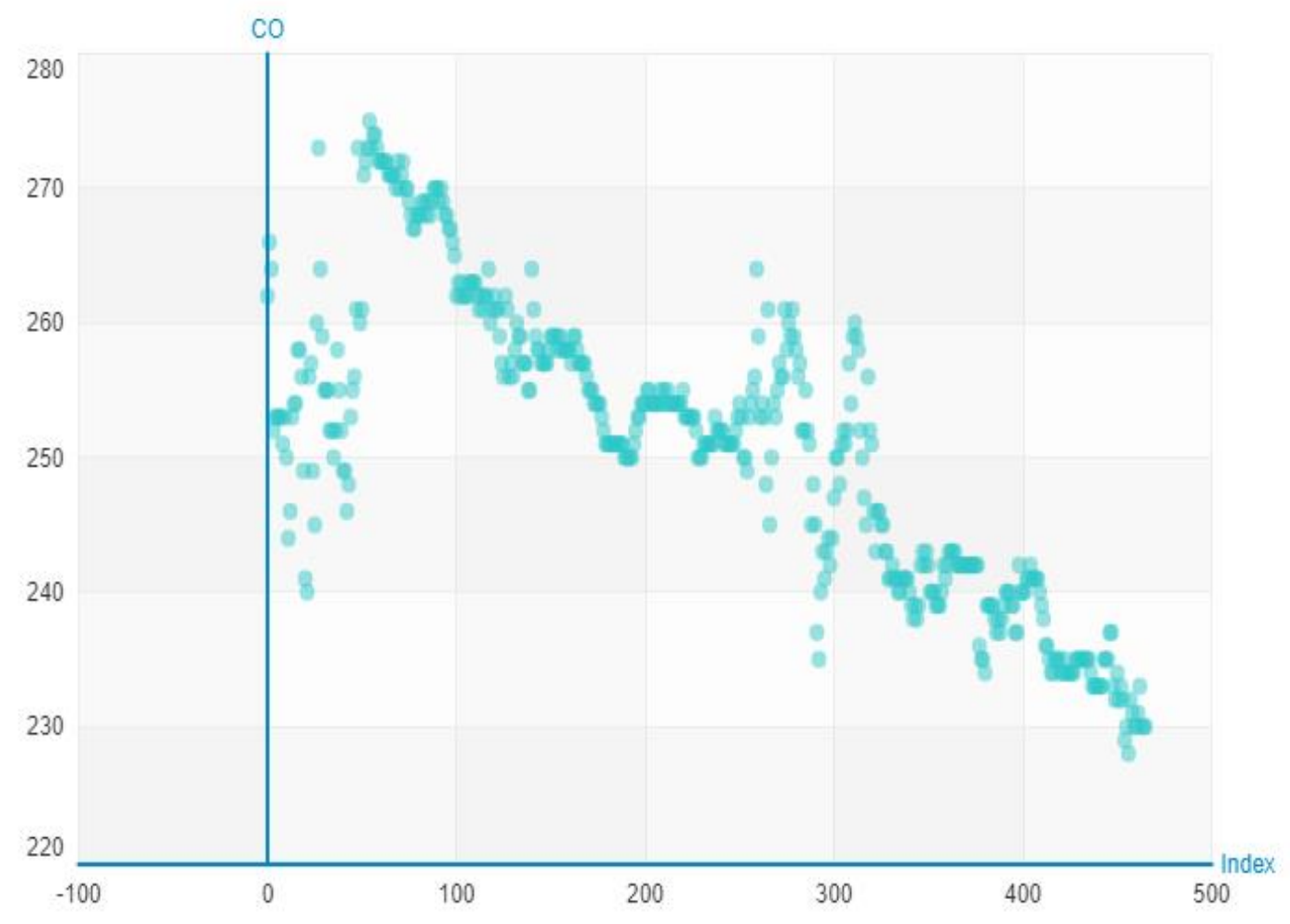

Fig-8: shows the variation of CO with Time (Index) in ScatterPlot. 


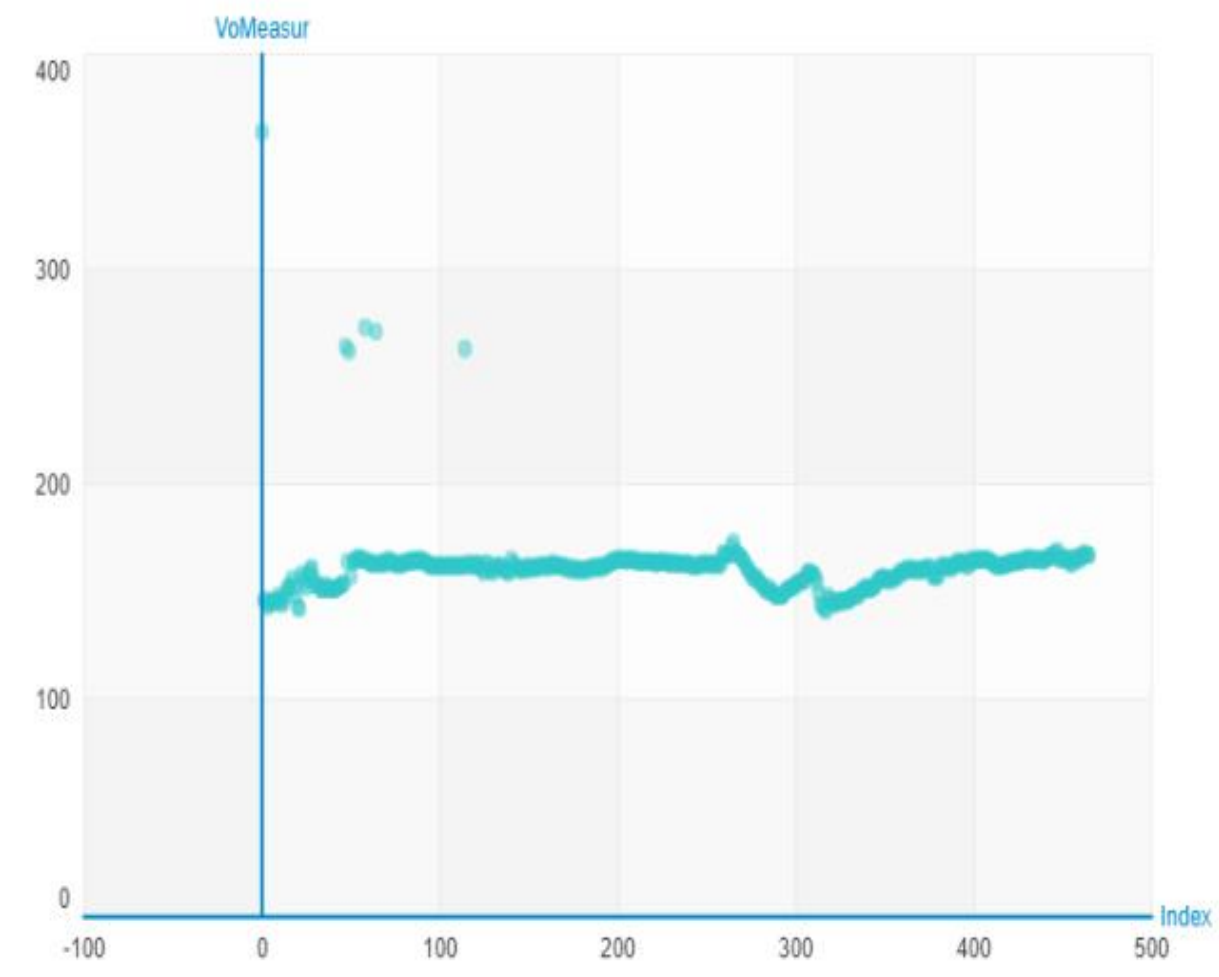

Fig-9: shows the variation of dust (VOMeasure) with Time (Index).
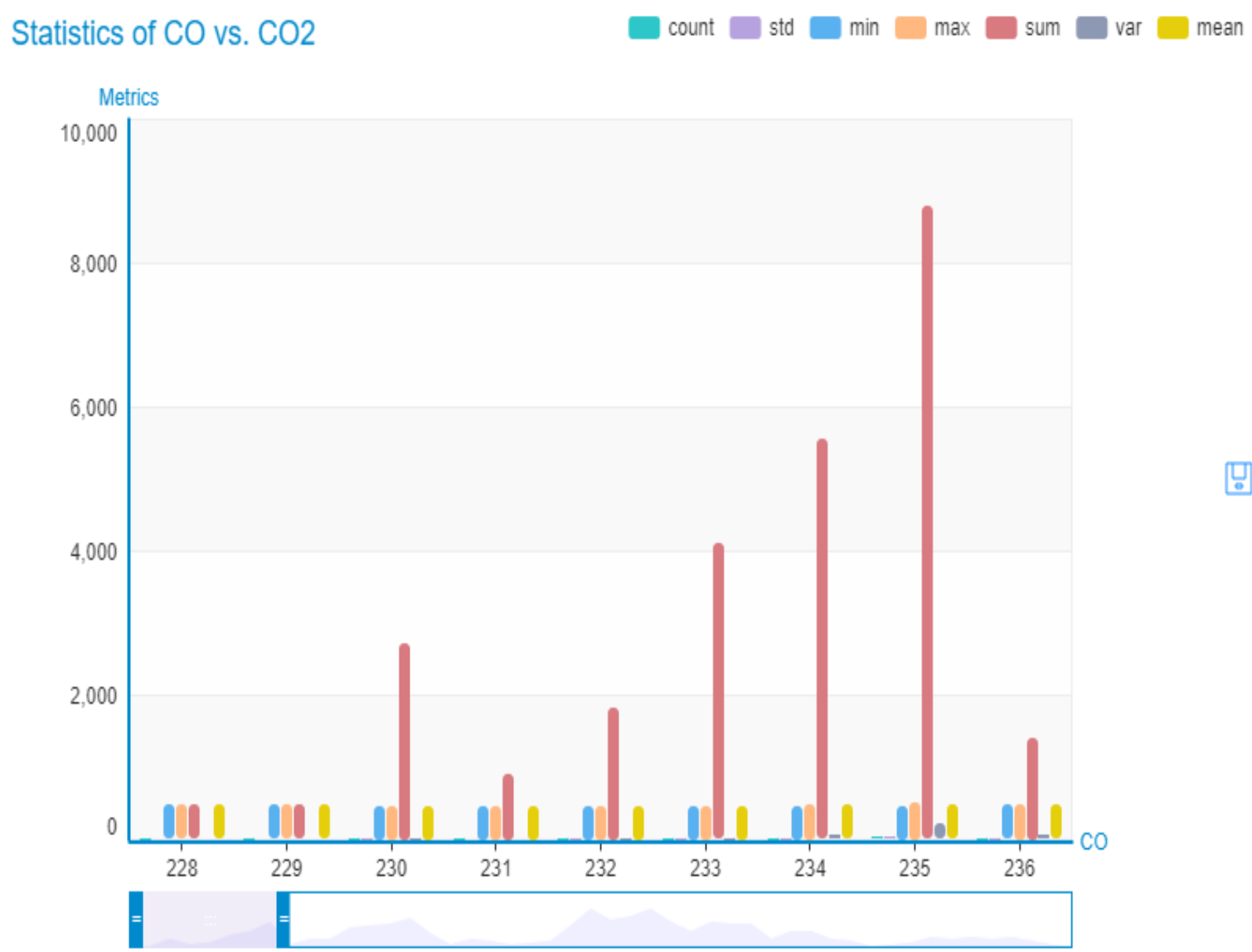

Fig-10: shows the Statistics of $\mathrm{CO}$ vs $\mathrm{CO}_{2}$ measure. 


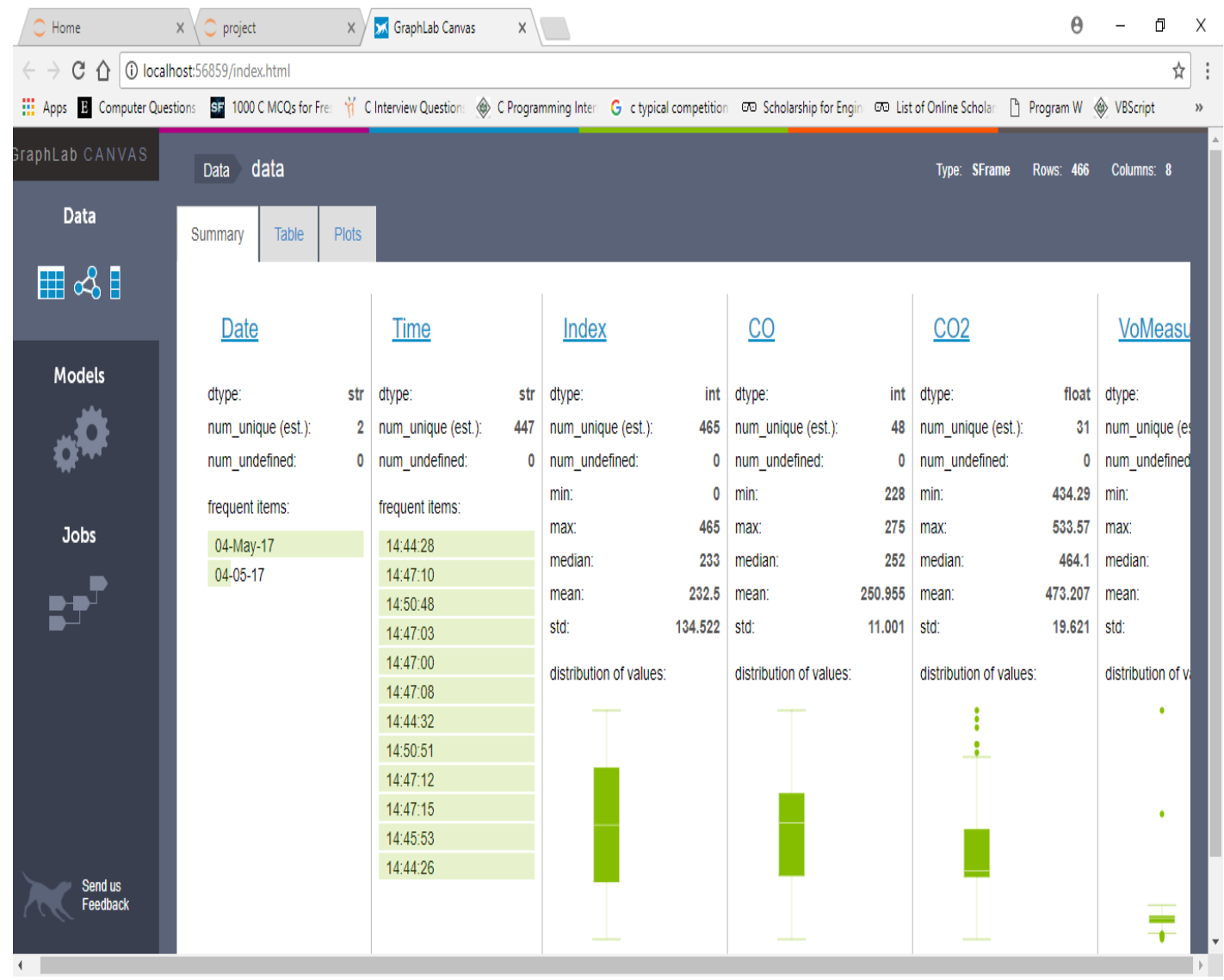

Fig-11: shows the Graphlab canvas of the dataset.

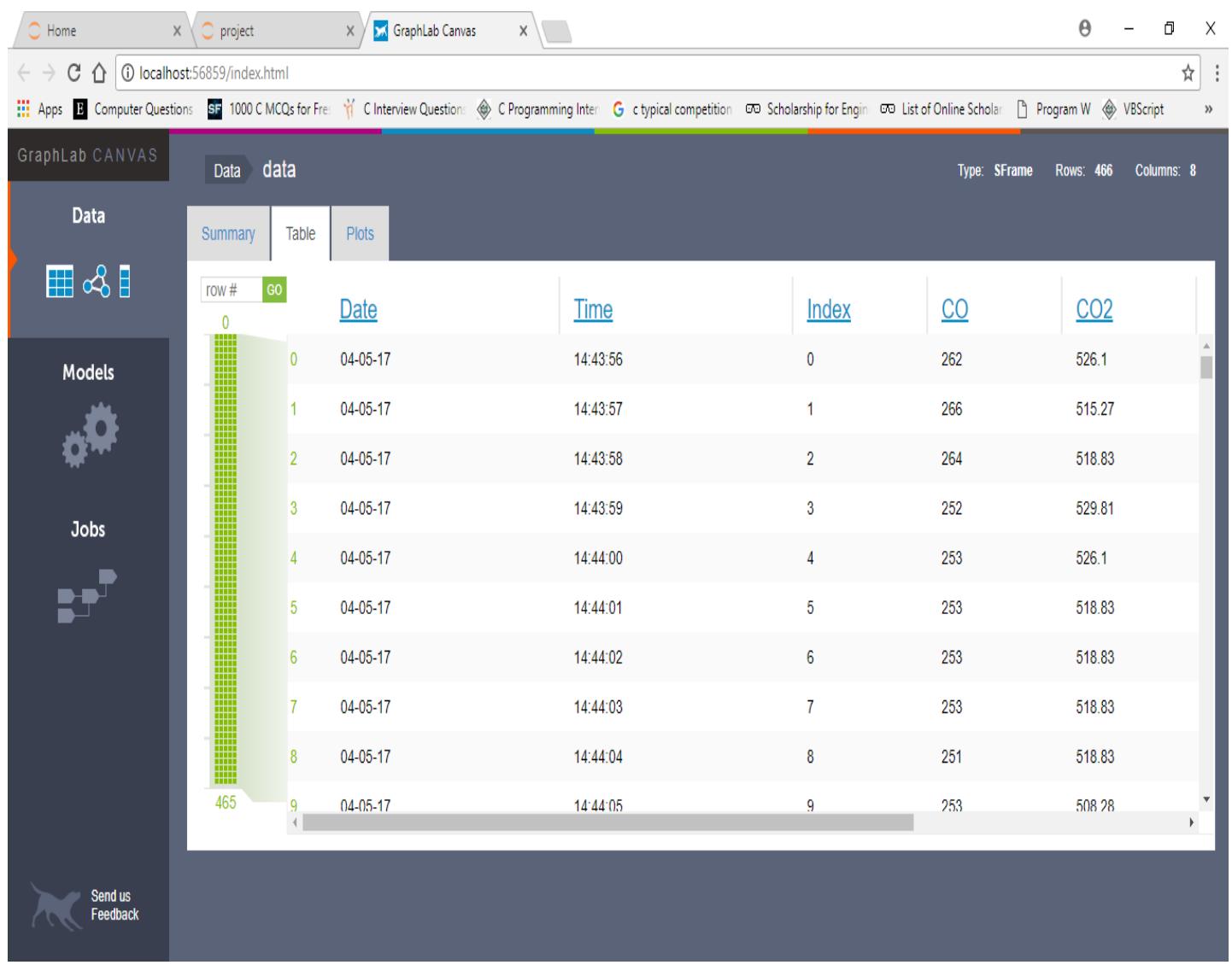

Fig-12: shows the datasheet. 


\section{FURTHER DEVELOPMENTS}

Further developments that can be done in this field that is, this IAQM kit can be installed in an HVAC system or in household air conditioning system so that it can monitor the air condition preset inside the room and then take several measures if the amount $\mathrm{CO}_{2}$ or dust present in the room is over adequate limits. This kit can also be implemented to build automatic air purification devices. Besides some notification system can be joined with it so that it can notify the user if the pollution quantity goes over a limit. The best result of this all is that system like this can help for the benefit of health by stopping and preventing the burden of symptoms and diseases linked with that of indoor air pollutants. In addition to this validation study, physical system and web portal improvements can be done by adding a real time feedback process to our android smartphone which is proposed in future for further development in AQM.

\section{CONCLUSION}

In this project work, we have developed an effective indoor air quality monitoring system with Raspberry pi microcontroller and specific sensors for collection of data. The main purpose was to show the show the changes for environmental parameters with persons which is shown effectively. The advantage of this model is its small size, low cost and high modularity; also the process of observation of data is easy and very user friendly. The result was obtained monitoring classrooms, bedrooms and other room with some persons and the obtained results were very promising giving a significant contribution to indoor air quality management.

\section{REFERENCES}

[1]. Erik Wilhelm, San.draSiby, Yuren Zhou, Xavier JayarajSiddarth Ashok, et al. , Wearable Environmental Sensors and Infrastructure for Mobile Large-Scale Urban Deployment ",IEEE SENSORS JOURNAL, VOL. 16, NO. 22, NOVEMBER 15, 2016.

[2]. Sean Dieter Tebje Kelly, Nagender Kumar Suryadevara, and Subhas Chandra Mukhopadhyay,"Towards the Implementation of IoT for Environmental Condition Monitoring in Homes" IEEE sensors journal, vol. 13, no. 10, October 2013.

[3]. Jung-Yoon Kim, Chao-Hsien Chu, and Sang-Moon Shin "ISSAQ: An Integrated Sensing. Systems for Real-Time Indoor Air Quality Monitoring" IEEE sensors journal, vol. 14, no. 12, december 2014.

[4]. Petros Spachos, Dimitrios Hatzinakos, "Real-Time Indoor Carbon Dioxide Monitoring Through Cognitive Wireless Sensor Networks," IEEE sensors journal, vol. 16, no. 2, january 15, 2016.

[5]. DivyaLohani and DebopamAcharya,"SmartVent: A Context Aware IoT System to Measure Indoor Air Qualityand Ventilation Rate," 2016 17th IEEE International Conference on Mobile Data Management.

[6]. Khaled Bashir Shaban, Senior Member, IEEE, Abdullah Kadri, Member, IEEE, and EmanRezk, "Urban Air Pollution

Monitoring System With Forecasting Models", IEEE SENSORS JOURNAL, VOL. 16, NO. 8, APRIL 15, 2016. [7]. Yifei Jiang, Kun Liz, LeiTian, Ricardo Piedrahita, Xiang Yun et al, "MAQS: A Personalized Mobile Sensing System for Indoor Air Quality Monitoring", UbiComp'11 / Beijing, China

[8]. Xuxu Chen, Yu Zheng, Yubiao Chen et al," Indoor Air Quality Monitoring System for Smart Buildings", UBICOMP '14, SEPTEMBER 13 - 17, 2014, SEATTLE, WA, USA.

[9]. H. Ghayvat , S.C. Mukhopadhyay, X. Gui, "Issues and mitigation of interference, attenuation and direction of arrival in IEEE 802.15.4/ZigBee to wireless sensors and networks basedsmart building", Measurement 86 (2016) 209-226.

[10]. PetrosSpachos, Liang Song, and DimitriosHatzinakos," Prototypes of Opportunistic Wireless Sensor Networks Supporting Indoor Air Quality Monitoring', The 10th Annual IEEE Consumer Communications and Networking Conference - Demos.

[11]. George Mois, TeodoraSanislav, and Silviu C. Folea, "A Cyber-Physical System for Environmental Monitoring", IEEE TRANSACTIONS ON INSTRUMENTATION AND MEASUREMENT, VOL. 65, NO. 6, JUNE 2016.

[12]. Yun Xiang, Ricardo Piedrahita, Robert P. Dick, Michael Hannigan, Qin Lv, Li Shang," A Hybrid Sensor System for Indoor Air Quality Monitoring", 2013 IEEE International Conference on Distributed Computing in Sensor Systems.

\section{BIOGRAPHIES}

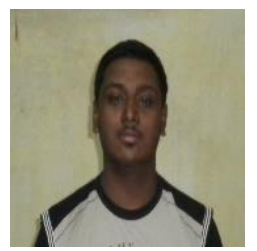

Somdeep Acharyya, $3^{\text {rd }}$ year Student of Dept. of Computer Science and Engineering in University Institute of Technology, Burdwan University pursuing B.E. degree.

\section{Email id: somdeep.acharyyasomdeep@gmail.com}
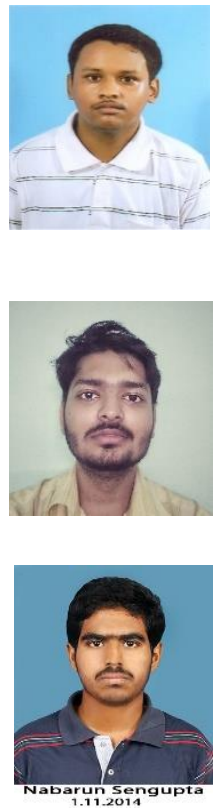

Email id: nabarunsengupta022@gmail.com
Sayan Hazra, $3^{\text {rd }}$ year Student of Dept. of Electrical Engineering in University Institute of Technology, Burdwan University pursuing B.E. degree. Email id:hazrasayan201442@gmail.com

Atanu Pal, $3^{\text {rd }}$ year Student of Dept. of Applied Electronics and Instrumentation Engineering in University Institute of Technology, Burdwan University pursuing B.E. degree.

\section{Email id:atanupal96@gmail.com}

Nabarun Sengupta, $3^{\text {rd }}$ year Student of Dept. of Electronics and Communication Engineering in University Institute of Technology, Burdwan University pursuing B.E. degree. 